\title{
Immunotherapy generates selective pressure for acquisition of immunogenic neoantigens in escape tumors
}

\author{
Jenny H Pan ${ }^{1 *}$, Suman Vodnala ${ }^{1}$, Robert L Eil ${ }^{1}$, Zhiya Yu', Jared J Gartner ${ }^{1}$, David Clever ${ }^{2}$, Rahul Roychoudhuri ${ }^{1}$, \\ Shashank J Patel ${ }^{2}$, Christopher A Klebanoff ${ }^{2}$, Madhusudhanan Sukumar ${ }^{2}$, Tori Yamamoto², Nicholas P Restifo ${ }^{1}$ \\ From 30th Annual Meeting and Associated Programs of the Society for Immunotherapy of Cancer (SITC 2015) \\ National Harbor, MD, USA. 4-8 November 2015
}

Host immunosurveillance as a mechanism to promote tumor growth or to suppress tumor development is well studied. It is less known how immuno-selective pressure in the form of immunotherapies can instruct the immune system during this process. We hypothesized that the application of a $\mathrm{T}$ cell-dependent selection pressure in the form of a whole tumor vaccine would alter the immunogenic architecture in our transplantable murine melanoma model SB-3123, and generated an escape variant to test this hypothesis.

We performed whole-exome and RNA-sequencing of the tumor line SB-3123 and two fresh tumors generated by subcutaneous implantation of SB-3123 to identify mutations and to assess levels of expression. There were 349 mutations shared between the tumor line and fresh tumors, with less than 5 mutations that were unique to each sample. We then determined the stability of these mutations and created a re-derived tissue culture line from a fresh SB-3123 tumor. Remarkably, the re-derived tissue culture line retained 333 mutations in common with the prior samples and produced only 2 unique mutations.

To test the immunogenicity of SB-3123, we vaccinated mice with irradiated SB-3123 and administered a live tumor challenge two weeks after vaccination. This resulted in a profound vaccination response and mice were completely protected from tumor for over 200 days. Surprisingly, one vaccinated mouse developed a recrudesced tumor at the site of implantation 40 days after challenge, and a tumor line was created (SB-3123-esc). We asked whether SB-3123-esc represented an antigen escape variant by immunizing mice with SB-3123 and challenging with either SB-3123 or SB-3123-esc. Vaccination with SB-3123 did not protect against SB-3123-esc. We also performed sequencing on SB-3123-esc and determined that no known genes involved in antigen processing and presentation were mutated, and SB-3123-esc upregulated class I molecules at similar levels compared to SB-3123 upon IFN- $\gamma$ treatment.

Our most striking results were the differences in mutational constituents between SB-3123 and SB-3123-esc. Of the nonsynonymous mutations called in the coding region, 441 were common to both tumor lines while 80 were unique to SB-3123 and 40 to SB-3123-esc. Furthermore, we were able to determine that SB-3123-esc lost 2 immunogenic neoantigens but had acquired 5 . Future investigations are directed towards identifying the rejection antigen responsible for the escape phenotype of SB-3123-esc.

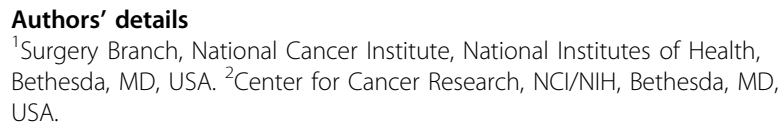

Published: 4 November 2015

\section{doi:10.1186/2051-1426-3-S2-P68}

Cite this article as: Pan et al:: Immunotherapy generates selective pressure for acquisition of immunogenic neoantigens in escape tumors. Journal for ImmunoTherapy of Cancer 2015 3(Suppl 2):P68. 